\title{
Severity Five Out of Five
}

National Cancer Institute

\section{Source}

National Cancer Institute. Severity Five Out of Five. NCI Thesaurus. Code C147510.

A severity result of five, on a five level scale. 